\title{
A review on gesture recognition technology in children's interactive storybook
}

\begin{abstract}
Over the past few years, gesture recognition has made its debut in education and virtual reality environment. This paper reviews the current literature in gesture recognition technology for interactive storybooks and the existing methods and challenges for this technology. A conceptual framework is proposed to resolve two main challenges that have been reviewed from previous work: to provide a novel interaction to young children and to ensure accuracy of gesture when using gesture based input sensor. The proposed method is the future work which provides the direction towards developing virtual reality storybooks for children.
\end{abstract}

Keyword: Children; Gesture recognition; Interactive storybooks; Leap motion controller 